\title{
Morbilidad postoperatoria en pacientes con hidatidosis hepática complicada
}

\author{
Carlos Manterola y Sebastián Urrutia, Grupo MINCIR
}

\section{Post surgery morbidity in patients with complicated hepatic hydatidosis}

Introduction: Hepatic echinococcosis (HE) surgery is common in southern's Chile hospitals; however, related publications are scarce and with conflicting results, especially with regard to postoperative morbidity (POM), due to the diversity in it severity. The aim of this study is to determine POM in patients undergoing surgery for complicated HE (CHE). Material and Methods: Case series part of a prospective cohort. Patients undergoing surgery for $\mathrm{CHH}$ in hospitals Hernán Henríquez Aravena hospital and Clínica Mayor of Temuco, between 2000 and 2012 were included. The main outcome variable was development of POM using the Clavien scale. Other variables of interest were hospital stay, mortality and recurrence. Descriptive statistics were applied and incidence of POM was determined. Results: 73 patients with a median age of 41 years and $58.9 \%$ female were studied. The median ultrasound diameter cysts were $15.0 \mathrm{~cm}$. The average surgical time was $125.4 \pm 26.3 \mathrm{~min}$. The surgical procedure used most often was the pericystectomy $(84.9 \%)$; and $84.9 \%$ of cases was performed concomitantly some other surgical procedure. The incidence of POM was $19.2 \%, 78.6 \%$ of whose cases were Grade I or II Clavien. The aetiology was $10.9 \%$ of medical complications and $8.2 \%$ of surgical complications. $1.4 \%$ of mortality was recorded; and with a median follow up of 97 months, no recurrence was verified. Conclusions: POM in a cohort of patients with CHE is lower than the published and low severity. Subgroups with higher POM were: patients with coexistence of more than one complication, those with colangiohydatidosis and patients with liver abscess of hydatid origin.

Key words: Echinococcosis, hydatidosis, echinococcosis, hepatic hydatid cyst, morbidity, cohort studies, prognosis

Palabras clave: Hidatidosis, quiste hidatídico hepático, morbilidad, estudios de cohorte, pronóstico.

\section{Introducción}

a enfermedad hidatídica es una zoonosis endémica en el sur de Chile, lo que se asocia no sólo con el tratamiento de numerosos casos nuevos de hidatidosis hepática $(\mathrm{HH})$ por año ${ }^{1}$, y con el hallazgo de enfermedad hidatídica en otras localizaciones abdomina$\mathrm{les}^{2}$; sino que además, con la pesquisa de complicaciones evolutivas de la $\mathrm{HH}$ y la necesidad de darle un tratamiento oportuno y eficiente.

Definimos como complicaciones evolutivas de la $\mathrm{HH}$ a la existencia de $\mathrm{HH}$ asociada a absceso hepático $(\mathrm{AHH})^{3,4}$, tránsito hepato-torácico $(\mathrm{THT})^{5}$, colangio-hidatidosis $(\mathrm{CH})^{6}$, peritonitis hidatídica ${ }^{2}$, anafilaxia, fistulización del quiste a alguna estructura del tubo digestivo; o la co-existencia de más de una ellas.

Siendo aún un problema de salud prevalente en algunas zonas del planeta, la literatura científica referente a cirugía de la HH no es tan numerosa, persistiendo cierta confusión y controversia en relación a la morbilidad de la cirugía de la HH. Los reportes de esta variable no han variado sustancialmente a lo largo del tiempo, independientemente del lugar de generación de los datos. En artículos de la década de los noventa se notificaron cifras de morbilidad postoperatoria (MPO) entre 34,7 y $62,5 \% \%^{7-13}$. Después del año 2000 la realidad no mejoró sustancialmente, pues se publicaron cifras de MPO de 21,3 a 53,8\% ${ }^{14-18}$; situación que podría explicarse por la heterogeneidad de las poblaciones analizadas en los diversos estudios, en los que suelen incluirse pacientes con $\mathrm{HH}$ complicada y no complicada; así como diferentes formas de valoración de la MPO.

En nuestro país, la magnitud de la hidatidosis humana y la situación epidemiológica se ha reportado con información proveniente de egresos hospitalarios y de notificación obligatoria entre los años 2001 y $2005^{19}$. En el citado estudio, se verificó que las tasas de incidencia en el período estudiado, obtenidas a partir de notificaciones y egresos (2,2/100.000 habitantes y 6/100.000 habitantes respectivamente), no habían variado a pesar de los esfuerzos sanitarios realizados (1.707 notificaciones y 4.742 egresos hospitalarios en el período). Se identificó a la Región de la Araucanía (donde se generan los datos del presente estudio) como una de las más afectadas (tasa pro-
Universidad de La Frontera Departamento de Cirugía (CM, SU) Centro de Excelencia en estudios Morfológicos y Quirúrgicos (CEMyQ, CM).

Universidad Autónoma de Chile. Centro de Investigación en Ciencias Biomédicas

Recibido: 28 de mayo de 2014 Aceptado: 11 de diciembre de 2014

Correspondencia a: Carlos Manterola Delgado carlos.manterola@ufrontera.cl 
medio de egresos de 28,1/100.000 habitantes), superada en este ítem sólo por la Región de Aysén con una tasa anual promedio de 43,8/100.000 habitantes ${ }^{19}$. Un artículo más reciente, que se basó en información recolectada a partir de datos del Sistema de Notificación Obligatoria (20012009), Egresos hospitalarios (2001-2008), Defunciones (2001-2008) y Años de Vida Potencialmente Perdidos (2001-2008); reportó que la incidencia promedio (según

\section{Tabla 1a. Clasificación de Clavien y cols. ${ }^{22,23}$}

\begin{tabular}{|c|c|}
\hline Grado & Definición \\
\hline I & $\begin{array}{l}\text { Cualquier desviación del curso post-operatorio normal que no requiera tratamiento far- } \\
\text { macológico, endoscópico, quirúrgico o de radiología intervencionista. Se permite apli- } \\
\text { cación de tratamientos farmacológicos como antieméticos, antipiréticos, analgésicos, } \\
\text { soluciones electrolíticas y fisioterapia. Incluye infección del sitio operatorio superficial } \\
\text { tratable en la cama del paciente }\end{array}$ \\
\hline$\|$ & $\begin{array}{l}\text { Se requiere de tratamiento farmacológico con medicamentos diferentes a los anteriores. } \\
\text { Incluye transfusión de hemoderivados y nutrición parenteral total }\end{array}$ \\
\hline $\begin{array}{l}\text { III } \\
\mathrm{b}\end{array}$ & $\begin{array}{l}\text { Requiere intervención quirúrgica, endoscópica o radiológica } \\
\text { Sin anestesia general } \\
\text { Con anestesia general }\end{array}$ \\
\hline $\begin{array}{l}\text { IV } \\
\text { a } \\
\text { b }\end{array}$ & $\begin{array}{l}\text { Complicación que amenaza la vida y que requieren tratamiento en unidades de cuida- } \\
\text { dos intermedios o intensivos } \\
\text { Disfunción de orgánica única (incluye utilización de diálisis) } \\
\text { Disfunción de orgánica múltiple }\end{array}$ \\
\hline V & Muerte del paciente \\
\hline Sufijo "d" & $\begin{array}{l}\text { Si el paciente sufre una complicación al momento del alta, al grado respectivo se agrega } \\
\text { el sufijo "d" (disability). Esto, indica la necesidad de seguimiento para una correcta } \\
\text { evaluación de la complicación }\end{array}$ \\
\hline
\end{tabular}

Nota: Adaptación del texto original.

\section{Tabla 1b. Ejemplos de los diferentes grados de la clasificación de Clavien y cols. .22,23 $^{2}$}

$\begin{array}{ll}\text { Grado } & \text { Ejemplos de situaciones clínicas } \\ \text { I } & \text { Infección del sitio quirúrgico superficial. Diarrea no infecciosa. Atelectasia pulmonar } \\ \text { que requiere fisioterapia } & \text { Infección urinaria que requiere antimicrobianos. Diarrea que requiere antimicrobianos. } \\ & \text { Neumonía tratada con antimicrobianos } \\ \text { III a } & \text { Cierre de herida dehiscente no infectada con anestesia local. Bilioma que requiere dre- } \\ & \text { naje percutáneo. Bradiarritmia que requiere marcapasos con anestesia local } \\ \text { III b } & \begin{array}{l}\text { Infección del sitio operatorio que conduce a eventración. Fístula anastomótica que } \\ \text { requiere re-laparotomía. Taponamiento cardíaco que requiere ventana pericárdica }\end{array} \\ \text { IV a } & \begin{array}{l}\text { Insuficiencia renal aguda que requiere diálisis. Falla respiratoria que requiere intubación. } \\ \text { IV b }\end{array} \\ & \begin{array}{l}\text { Falla respiratoria que requiere intubación asociada a falla renal. Accidente cerebro } \\ \text { vascular con falla respiratoria. Falla cardiaca que conduce a síndrome de bajo débito } \\ \text { en combinación con falla renal }\end{array}\end{array}$

notificaciones obligatorias) fue 1,9/100.000 habitantes, la tasa promedio de egresos hospitalarios fue de 6,3/100.000 habitantes y la tasa de mortalidad promedio fue 0,2 defunciones por 100.000 habitantes, siendo las Regiones de Aysén y la Araucanía las más afectadas ${ }^{20}$.

El objetivo de este estudio es determinar la incidencia de MPO en pacientes intervenidos quirúrgicamente por HH complicada.

\section{Material y Método}

\section{Tipo de estudio}

Serie de casos de pacientes intervenidos por $\mathrm{HH}$ complicada, que son parte de una cohorte prospectiva de sujetos con $\mathrm{HH}$ intervenidos quirúrgicamente.

\section{Población}

Se incluyeron pacientes operados por HH complicada, en los Servicios de Cirugía del hospital Hernán Henríquez Aravena y de la Clínica Mayor de la ciudad de Temuco, Chile, en el período mayo de 2000 y mayo de 2012, por uno de los autores (CM). Se excluyeron pacientes intervenidos por vía laparoscópica, sujetos con hidatidosis concomitante en otra localización y pacientes intervenidos previamente por $\mathrm{HH}$.

\section{Protocolo de estudio}

Se confeccionó una hoja de recolección de datos en las que se registraron las características biodemográficas y clínicas de los pacientes, los hallazgos de laboratorio e imágenes ${ }^{21}$, las características de los quistes, los aspectos quirúrgicos relacionados; y la evolución postoperatoria en términos de MPO, necesidad de re-intervenciones, mortalidad y recurrencia.

\section{Variables de interés}

La variable resultado fue el desarrollo de MPO, la que se trabajó de forma dicotómica (sí/no), categorizando por origen (ninguna/médica/quirúrgica) y por gravedad, aplicando la escala de Clavien ${ }^{22,23}$ (Tabla 1); para ello se exigió un seguimiento mínimo de 12 meses. Otras variables de interés fueron estancia hospitalaria, necesidad de re-intervenciones, mortalidad y recurrencia.

\section{Protocolo de seguimiento}

Se realizó control clínico con laboratorio general, determinación de inmunoglobulinas específicas (ELISAIgG y ELISA-IgE), radiología de tórax y ultrasonografía abdominal en los meses 1, 6 y 12; y, de ahí en adelante, una vez por año del post-operatorio, por un mínimo de cuatro años.

\section{Tratamiento realizado}

No hubo un tratamiento estándar, pues los procedimientos quirúrgicos empleados se aplicaron de acuerdo a 
los hallazgos intraoperatorios (tipo de complicación evolutiva). Según éstos, se realizó periquistectomía o resecciones hepáticas (lobectomías o hepatectomías atípicas), asociadas a algún otro procedimiento complementario.

\section{Plan de análisis}

Se realizó un análisis exploratorio de los datos. Posteriormente, se utilizó estadística descriptiva con cálculo de porcentajes, medidas de tendencia central y dispersión (promedios y desviación estándar; mediana y valores extremos), dependiendo de la normalidad de la distribución de los datos. Finalmente, se calculó incidencia o riesgo absoluto para el desarrollo de MPO.

\section{Resultados}

En el período estudiado fueron tratados según este esquema 73 pacientes con $\mathrm{HH}$ complicada, sin registrarse pérdidas de seguimiento. El flujograma de los participantes se detalla en la Figura 1. La mediana de edad fue de 41 años (16 a 84 años); y 58,9\% de los pacientes (43 casos), era de género femenino.

Veinticinco pacientes $(34,3 \%)$ eran portadores de alguna patología asociada, entre las que destaca la colelitiasis (13 casos, 17,8\%) (Tabla 2).

Los 73 sujetos en estudio presentaban un total de 114 quistes de diverso tamaño y localización. La mediana del diámetro ultrasonográfico de la totalidad de los quistes fue de 15,0 cm (6 a $30 \mathrm{~cm}$ ). Las lesiones se localizaron preferentemente en el lóbulo derecho $(72,6 \%)$ y eran mayoritariamente de patrón ultrasonográfico CL4 (28,8\% de los casos). La distribución del resto de las lesiones, se aprecia en la Tabla 3.

En la mayor parte de los casos no se pudo realizar medición inicial de inmunoglobulinas específicas, pues fueron intervenidos de urgencia. ELISA-IgG específica fue positiva en 14 de los 17 pacientes en los que se logró medir ( $82,4 \%$ de positividad); y ELISA-IgE específica fue positiva en 7 de los 17 pacientes en los que se logró medir (41,2\% de positividad). El seguimiento con inmunoglobulinas específicas en estos casos, permitió verificar que en $17,6 \%$ de los casos, ELISA-IgE se mantuvo negativa; y en los casos inicialmente positivos, se observó un porcentaje de negativización de 57,1 y 42,9\% a los dos y cuatro años, respectivamente. El comportamiento de ELISA-IgG fue similar: en 21,4\% de los casos, se mantuvo negativo; y se verificó negativización en 42,9 y 35,7\% a los dos y cuatro años, respectivamente.

El tiempo quirúrgico promedio fue de 125,4 $\pm 26,3$ min, y el procedimiento quirúrgico utilizado con mayor frecuencia fue la periquistectomía $(84,9 \%)$. El 27,4\% de los pacientes tenían dos o más quistes y 78,9\% de los quistes principales tenían comunicaciones biliares evidentes. Por otra parte, en $84,9 \%$ de los casos se realizó de forma concomitante algún otro procedimiento quirúrgico (Tabla 3).

Se verificó la existencia de las siguientes complicaciones evolutivas como hallazgos únicos: $\mathrm{AHH}$ en 34 casos (46,5\%), $\mathrm{CH}$ en 11 casos $(15,1 \%)$, THT en ocho casos $(11,0 \%)$, peritonitis hidatídica en cuatro casos $(5,5 \%)$, y anafilaxia en tres casos $(4,1 \%)$. Se observó además, la co-existencia de más de una complicación evolutiva en 13 casos (17,8\%). Estos; corresponden a dos casos de $\mathrm{AHH}$

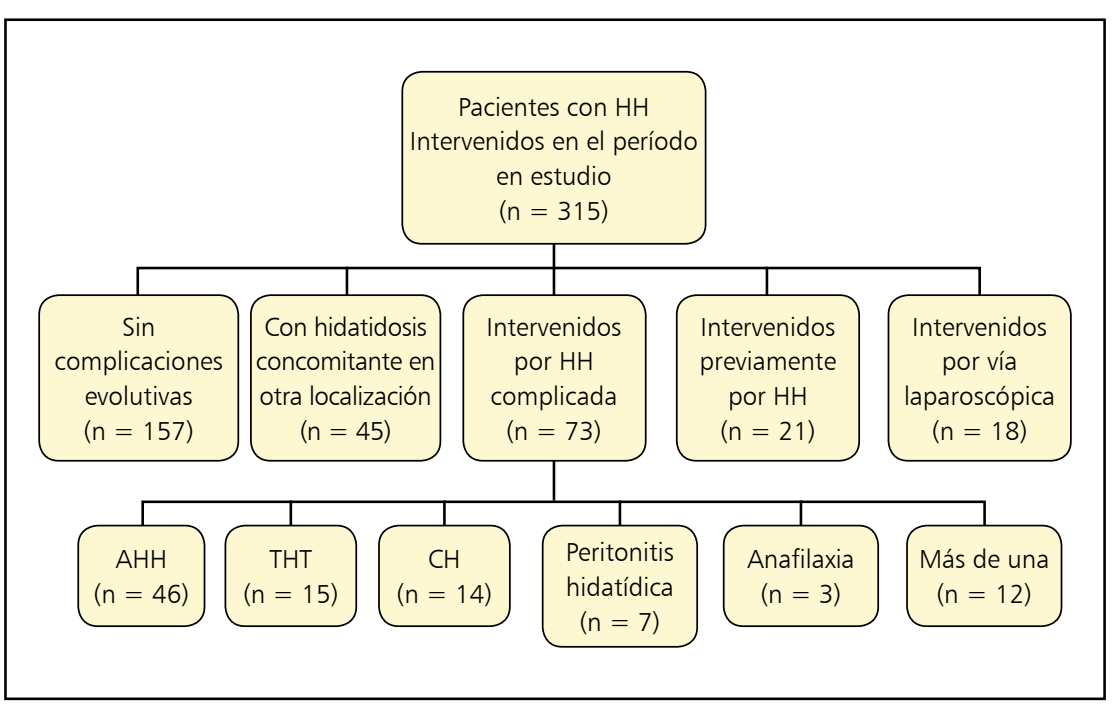

Figura 1. Flujograma de los participantes del estudio.

Tabla 2. Co-morbilidad asociada en los pacientes en estudio (n: 73)

\begin{tabular}{lcc} 
Variable & n casos & $\%$ \\
Patología asociada (\%) & 48 & 65,7 \\
Sin patología & 13 & 17,8 \\
Colelitiasis & 1 & 1,4 \\
LCFA & 4 & 5,6 \\
Cardiopatía & 1 & 1,4 \\
DM & 1 & 1,4 \\
Cardiopatía + DM & 1 & 1,4 \\
Desnutrición & 2 & 2,7 \\
Embarazo* & 1 & 1,4 \\
Trauma abdominal** & 1 & 1,4 \\
Cáncer gástrico*** & & \\
\hline
\end{tabular}

LCFA: Limitación crónica al flujo aéreo. DM: Diabetes mellitus tipo II. *Embarazo de 15 semanas. **La HH fue un hallazgo intraoperatorio pesquisado durante la laparotomía exploradora por trauma abdominal; y fue resuelto en el mismo tiempo quirúrgico. ***El cáncer gástrico fue un hallazgo intraoperatorio pesquisado durante la laparotomía exploradora; y fue resuelto en el mismo tiempo quirúrgico. 
Tabla 3. Distribución de algunas variables clínicas de los pacientes en estudio (variables categóricas) ( $\mathrm{n}: 73)$

\begin{tabular}{|c|c|c|}
\hline Variable & n casos & $\%$ \\
\hline \multicolumn{3}{|c|}{ Características ultrasonográficas del quiste* } \\
\hline \multicolumn{3}{|l|}{$\mathrm{CL}$} \\
\hline $\mathrm{CL} 1$ & 20 & 27,4 \\
\hline $\mathrm{CL2}$ & 12 & 16,4 \\
\hline $\mathrm{CL3}$ & 18 & 24,7 \\
\hline CL4 & 21 & 28,8 \\
\hline CL5 & 2 & 2,7 \\
\hline \multicolumn{3}{|l|}{ Localización del quiste* } \\
\hline Lóbulo derecho & 53 & 72,6 \\
\hline Lóbulo izquierdo & 8 & 11,0 \\
\hline Bilateral & 12 & 16,4 \\
\hline \multicolumn{3}{|l|}{$\mathrm{n}$ de quistes } \\
\hline Uno & 53 & 72,6 \\
\hline Dos o más & 20 & 27,4 \\
\hline \multicolumn{3}{|l|}{ Comunicaciones biliares* } \\
\hline Ninguna & 16 & 21,9 \\
\hline Una & 40 & 54,8 \\
\hline Dos o más & 17 & 23,3 \\
\hline \multicolumn{3}{|l|}{ Cirugía realizada en la lesión principal } \\
\hline Periquistectomía & 62 & 84,9 \\
\hline Resección hepática & 11 & 15,1 \\
\hline \multicolumn{3}{|c|}{ Procedimientos quirúrgicos concomitantes } \\
\hline Ninguno & 11 & 15,1 \\
\hline Colecistectomía & 35 & 47,9 \\
\hline Colecistectomía y coledocostomía & 20 & 27,4 \\
\hline Coledocostomía & 6 & 8,2 \\
\hline Gastrectomía & 1 & 1,4 \\
\hline
\end{tabular}

Tabla 4. Distribución de variables de laboratorio de los pacientes en estudio (n: 73)

\begin{tabular}{|lcccc|} 
Variable & Mediana & $\begin{array}{c}\text { Valores } \\
\text { extremos }\end{array}$ & Media \pm DE & $\begin{array}{c}\text { Valores de } \\
\text { referencia }\end{array}$ \\
Hematocrito (\%) & 38 & $22-50$ & $36,9 \pm 5,3$ & $41,0-49,0$ \\
Leucocitos $\left(\mathrm{mm}^{3}\right)$ & 9.600 & $3.900-32.400$ & $11.068,4 \pm 4.945,3$ & $4,1-9,5$ \\
Bilirrubinemia total (mg/dl) & 0,9 & $0,9-23,0$ & $2,0 \pm 3,1$ & $0,2-1,3$ \\
Fosfatasas alcalinas (U/L) & 420 & $69-2.323$ & $574,1 \pm 501,1$ & $38-126$ \\
ASAT (U/L) & 31 & $3-1.044$ & $75,6 \pm 132,7$ & $17-59$ \\
ALAT (U/L) & 37 & $6-738$ & $81,2 \pm 115,3$ & $21-72$ \\
\hline DE: Desviación estándar. & & & & \\
\hline
\end{tabular}

$+\mathrm{CH}$, seis casos de $\mathrm{AHH}+\mathrm{THT}$, tres casos de $\mathrm{AHH}+$ peritonitis hidatídica y dos casos de $\mathrm{AHH}+\mathrm{CH}+\mathrm{THT}$.

De los estudios de laboratorio realizados destaca el valor elevado de la mediana de la determinación de fosfatasas alcalinas (Tabla 4).

La mediana de estancia hospitalaria fue de cinco días (2 a 28 días). Con un seguimiento promedio de 97,6 \pm 51,2 meses, no se constató recurrencia. La incidencia o riesgo absoluto de MPO fue de 19,2\% (14 pacientes del total a lo largo de todo el período de seguimiento), 78,6\% de cuyos casos correspondieron a Grado I o II de Clavien. La etiología se desagrupó de la siguiente forma: ocho casos $(10,9 \%)$ de complicaciones de tratamiento médico (siete casos de atelectasia y uno de arritmia); y seis casos (8,2\%), de complicaciones de tratamiento quirúrgico (cuatro casos de infección de sitio operatorio, uno de fístula biliar y uno de eventración [estos dos últimos requirieron de una reintervención quirúrgica; lo que representa una incidencia de re-intervenciones de 2,7\%]) (Tabla 5).

La MPO desagrupada para los subgrupos de complicaciones evolutivas fue: $27,3 \% ; 17,6 \% ; 12,5 \% ; 0,0 \%$ y $0,0 \%$ para $\mathrm{CH}, \mathrm{AHH}, \mathrm{THT}$, peritonitis y anafilaxia, respectivamente; y de $30,8 \%$ en casos de co-existencia de más de una complicación evolutiva. Esto y la etiología de la MPO se resumen en la Tabla 6.

La mortalidad fue de 1,4\% (dos casos). Uno de los pacientes, de sexo femenino, 33 años, era portadora de un absceso hepático hidatídico y tránsito hepatotorácico simultáneo; y falleció a consecuencia de una falla respiratoria aguda de origen no precisado, a las $48 \mathrm{~h}$ del post-operatorio. Y el segundo paciente, también de sexo femenino, con 84 años de edad, era portadora de un absceso hepático hidatídico; y falleció a los 27 meses de seguimiento a consecuencia de una falla cardíaca de origen no precisado. 


\begin{tabular}{|c|c|c|c|c|c|}
\hline Subgrupo & n casos & $\%$ & Respiratorias & ISQ & Otras** \\
\hline $\mathrm{CH}$ & $3 / 11$ & 27,3 & $0 / 3$ & $1 / 3$ & $2 / 3$ \\
\hline $\mathrm{AHH}$ & $6 / 34$ & 17,6 & $2 / 6$ & $3 / 6$ & $1 / 6$ \\
\hline THT & $1 / 8$ & 12,5 & $1 / 1$ & $0 / 1$ & $0 / 1$ \\
\hline Peritonitis hidatídica & $0 / 4$ & 0,0 & $0 / 0$ & $0 / 0$ & $0 / 0$ \\
\hline Anafilaxia & $0 / 3$ & 0,0 & $0 / 0$ & $0 / 0$ & $0 / 0$ \\
\hline Coexistencia de más de una complicación evolutiva* & $4 / 13$ & 30,8 & $4 / 4$ & $0 / 4$ & $0 / 4$ \\
\hline Total & $14 / 73$ & 19,2 & $7 / 14$ & $4 / 14$ & $3 / 14$ \\
\hline
\end{tabular}

\section{Discusión}

Como se comentó anteriormente, las publicaciones relacionadas con la cirugía de la $\mathrm{HH}$ son relativamente escasas y de bajo nivel de evidencia; es así como al buscar en la base de datos MEDLINE, utilizando los términos MeSH "Echinococcosis, Hepatic/surgery"[Mesh], se obtuvieron 2.055 registros; los que al ser depurados a través de la aplicación del operador booleano NOT ("alveolar echinococcosis", "pulmonary echinococcosis", "lung echinococcosis", "pharmacological treatment", "laparoscopic surgery") y algunos límites (artículos publicados desde 2000 a la fecha de la revisión [26/05/2014], en población humana, adulta y en idiomas español e inglés), arrojó una cantidad de 399 artículos. Ahora bien, cuando a éstos le agregamos límites relacionados con el tipo de diseño de los artículos buscando el mejor nivel de evidencia (ensayos clínicos y revisiones sistemáticas), la búsqueda sólo generó un total de 19 artículos. Esto quiere decir, que en los últimos quince años, más de $95 \%$ de los artículos referentes a cirugía de la $\mathrm{HH}$ siguen siendo series de casos (en su gran mayoría de carácter retrospectivo); la mayor parte de las cuales contienen población heterogénea y por ende, con resultados cuya validez interna y externa es objetable. Por otra parte, al realizar una búsqueda en las bases de datos MEDLINE y PUBMED, con el término libre quiste hidatídico hepático complicado y algunos límites (artículos publicados desde 2000 a la fecha de la revisión [26/05/2014], en población humana, adulta; y en idiomas español, inglés), sólo se obtuvieron 49 artículos; pero 33 de ellos no tenían relación alguna con la frase quiste hidatídico complicado.

No obstante lo anteriormente expuesto, uno de los dilemas permanentes y que, a pesar del avance tecnológico persiste como un problema. es la MPO de la HH. Llama la atención el hecho que en los últimos siete años se sigan reportando cifras de 21,3 a $53,8 \%{ }^{16-18,24}$; sobre todo si éstas se miran desde la perspectiva de que se trata de una enfermedad benigna. Es posible que estos guarismos que parecen desproporcionados y dispersos se relacionen a la heterogeneidad de las poblaciones estudiadas pues, como ya se señaló, en ellos, se mezclan pacientes HH complicada y no complicada, intervenidos quirúrgicamente con técnicas muy diversas y en centros con disímil nivel de experiencia en este tipo de cirugía.

También parece interesante mencionar que la co-morbilidad objetivada (34,3\% de los casos), puede de alguna forma incidir en el desarrollo de MPO; entre otras cosas porque en algunos casos, fue el detonante de la necesidad de realizar cirugías adicionales (60,0\% de los casos).

La mediana de estancia hospitalaria, de cinco días contrasta con datos previamente publicados que fluctúan entre 6,5 y 23 días de promedio ${ }^{15,25-28}$.

La incidencia observada de MPO en pacientes intervenidos por $\mathrm{HH}$ complicada $(19,2 \%)$, nos parece adecuada en relación a estudios previos de $\mathrm{HH}$ complicada, respecto de lo cual se han publicado cifras entre 27,8 y $55,0 \%{ }^{26,28-30}$; porque la gravedad de las complicaciones observadas, aplicando la propuesta de Dindo \& Clavien $^{22,23}$, es mayoritariamente baja (sólo 30\% de los casos fue de resorte quirúrgico y por ende, requirieron una re-operación); y, porque la MPO reportada fue analizada a lo largo de un extenso período de seguimiento $(97,6$ meses), el que supera largamente a los reportes previos respecto del tema.

Por otra parte, la ausencia de recurrencia, asociada a lo extenso del período de seguimiento, también contrasta con cifras anteriores que oscilan entre 1,9 y $12,5 \%{ }^{17,24,29,31}$. 
En relación con el seguimiento de los pacientes, parece de interés comentar el comportamiento de las inmunoglobulinas específicas que se midieron a lo largo del tiempo en algunos pacientes; situación similar a la anteriormente reportada por nuestro grupo (negativización progresiva de ELISA-IgE y de ELISA-IgG, hasta alcanzar 98 y $84 \%$ al cuarto año de seguimiento, respectivamente, detectando además, un comportamiento errático de ELISA-IgG, con viraje de negativo a positivo en $11 \%$ de los casos, en ausencia de imágenes quísticas radiológicas o ecotomográficas en el tórax y el abdomen $)^{32}$.

La co-existencia de más de una complicación evolutiva (12 casos; 16,4\%), dificulta el conocer con fineza la incertidumbre respecto de la asociación entre algunas complicaciones evolutivas y la etiología de la MPO. Sin embargo, se puede comentar que el análisis de estas variables permitió, no sólo identificar los subgrupos con mayor MPO, sino además, la asociación de $\mathrm{AHH}$ e infección del sitio operatorio y de complicaciones respiratorias con sujetos en los que co-existía más de una complicación evolutiva.

Finalmente, la mortalidad registrada de 1,4\% (dos pacientes), es acorde a reportes previos en los que la mortalidad varía entre 0,0 y $2,6 \% 0^{17,27,28,30,31,33,34}$. En el análisis de esta variable, se ha de considerar que en uno de los casos, la paciente presentaba co-existencia de dos complicaciones evolutivas de la enfermedad; y que el otro caso, se trataba de una paciente de 84 años que falleció más de dos años posteriores a la cirugía.

A modo de conclusión, se describe la incidencia de MPO en un grupo de pacientes con $\mathrm{HH}$ complicada con énfasis en la gravedad de ésta. La MPO observada fue inferior a la publicada y de bajo nivel de gravedad y los subgrupos de complicaciones evolutivas con mayor MPO fueron la co-existencia de más de una complicación, la $\mathrm{CH}$ y el AHH.

\section{Resumen}

Introducción: La cirugía de la hidatidosis hepática (HH) es habitual en hospitales del sur de Chile; sin embargo, las publicaciones relacionadas son escasas y sus resultados disímiles, en especial respecto de la morbilidad postoperatoria (MPO), debido a la diversidad en la gravedad. El objetivo de este estudio es determinar la incidencia de MPO en pacientes intervenidos quirúrgicamente por HH complicada. Material y Método: Serie de casos, parte de una cohorte prospectiva. Se incluyeron pacientes intervenidos quirúrgicamente por HH complicada, en el hospital Hernán Henríquez Aravena y en la Clínica Mayor de Temuco, entre 2000 y 2012. La variable resultado principal fue desarrollo de MPO aplicando la escala de Clavien. Otras variables de interés fueron estancia hospitalaria, mortalidad y recurrencia. Se aplicó estadística descriptiva y, se determinó incidencia de MPO. Resultados: 73 pacientes, con mediana de edad de 41 años (16 a 84 años); 58,9\% de género femenino. La mediana del diámetro ultrasonográfico de los quistes fue de $15,0 \mathrm{~cm}$. El tiempo quirúrgico promedio fue de $125,4 \pm 26,3 \mathrm{~min}$. El procedimiento quirúrgico utilizado con mayor frecuencia fue periquistectomía $(84,9 \%)$; y en $84,9 \%$ de los casos se realizó de forma concomitante algún otro procedimiento quirúrgico. La incidencia de MPO fue $19,2 \%$ (10,9\% de tratamiento médico y $8,2 \%$ de tratamiento quirúrgico); 78,6\% de cuyos casos eran Grado I o II de Clavien. Se registró $1,4 \%$ de mortalidad; y con una mediana de seguimiento de 97 meses, no se verificó recurrencia. Conclusiones: La incidencia de MPO en pacientes con $\mathrm{HH}$ complicada es inferior a la publicada y de bajo nivel de gravedad. Los subgrupos de complicaciones evolutivas con mayor MPO fueron la co-existencia de más de una complicación, la colangiohidatidosis y el absceso hepático de origen hidatídico.

\section{Referencias bibliográficas}

1.- Servicio de Cirugía Hospital Hernán Henríquez Aravana de Temuco. Servicio de Salud Araucanía Sur. Estadísticas quirúrgicas año 2012.

2.- Manterola C, Vial M, Losada H, Fonseca F, Bustos L, Muñoz S, et al. Uncommon locations of abdominal hydatid disease. Trop Doct 2003; 33: 179-80.

3.- Manterola C, Barroso M, Vial M, Bustos L, Muñoz S, Losada H, et al. Liver abscess of hydatid origin: clinical features and results of aggressive treatment. ANZ J Surg 2003; 73 : 220-4.

4.- Manterola C, Sanhueza A, Vial M, Moraga J, MINCIR Group. Liver abscess of hydatid origin as a risk factor for postoperative complications in hidatidosis. Rev Chil Cir 2009; 61: 333-8.

5.- Manterola C, Ávila N, Seco J, Ulloa P, Moraga J. Grupo MINCIR. Hepatothoracic transit, evolutionary complications of hepatic echinoccocosis. Rev Chil Cir 2009; 61: 345-9.

6.- Manterola C, Losada H, Carrasco R, Muñoz S, Bustos L, Vial M, Innocenti G. Cholangiohydatidosis. An evolutive complication of hepatic hydatidosis. Bol Chil Parasitol 2001; 56: 10-5.

7.- Pinto P. Hidatidosis hepática: estudio de una serie de 534 casos. Rev Chil Cir 1991; 43: 184-7.

8.- Apablaza S, Burmeister R, Benavides, C. Tratamiento del quiste hidatídico hepático.
Rev Chil Cir 1992; 44: 424-7.

9.- Vallejos C. Postbeca en región de hidatidosis endémica. Rev Chil Cir 1994; 46: 414-7.

10.- Camacho J, Reyes J Pérez A. Cirugía del quiste hidatídico hepático. Rev Chil Cir 1996; 48: 479-82.

11.- González D, Vega A, Monti J, Torres M. Enfermedad hidática hepática: experiencia de los últimos 13 años en Florida. Cir Urug 1999; 69: 200-3.

12.- Sarotto L, Nallar M, Ferraro A, Danguise E, Merello J, Ferraina P. Tratamiento quirúrgico de la hidatidosis hepática: experiencia en el Hospital de Clínicas en los últimos 15 años. Rev Argent Cir 1999; 76: 94-105.

13.- Correa S, Culqui C, Pinto M. Hidatidosis hepática: revisión de casos intervenidos 
quirúrgicamente en el Hospital Militar Central Lima 1990-1999. Rev Gastroenterol Perú 2000; 20: 261-9.

14.- Nari G, Ponce O, Cirami M, Jozami J, Toblli J, Eduardo M, Fernando M. Five years experience in surgical treatment of liver hydatidosis. Int Surg 2003; 88: 194-8.

15.- Atmatzidis K S, Pavlidis T E, Papaziogas B T, Mirelis C, Papaziogas T B. Recurrence and long-term outcome after open cystectomy with omentoplasty for hepatic hydatid disease in an endemic area. Acta Chir Belg 2005; 105: 198-202.

16.- Daradkeh S, El-Muhtaseb H, Farah G, Sroujieh A S, Abu-Khalaf M. Predictors of morbidity and mortality in the surgical management of hydatid cyst of the liver. Langenbecks Arch Surg 2007; 392: 35-9.

17.- Gourgiotis S, Stratopoulos C, Moustafellos P, Dimopoulos N, Papaxoinis G, Vougas V, et al. Surgical techniques and treatment for hepatic hydatid cysts. Surg Today 2007; 37: 389-95.

18.- Agayev R M, Agayev B A. Hepatic hydatid disease: surgical experience over 15 years. Hepatogastroenterology 2008; 55: 1373-9.

19.- Cortés S, Valle C. Human hydatidosis: general aspects and epidemiological situation in Chile according to hospital discharge and mandatory reporting from 2001 to 2005 . Rev Chilena Infectol 2010; 27: 329-35.

20.- Martínez P. Human hydatidosis disease: general background and epidemiological situation in
Chile, 2001-2009. Rev Chilena Infectol 2011; 28: 585-91.

21.- WHO Informal Working Group. International classification of ultrasound images in cystic echinococcosis for application in clinical and field epidemiological settings. Acta Trop 2003; 85: 253-61.

22.- Dindo D, Demartines N, Clavien P A. Classification of surgical complications a new proposal with evaluation in a cohort of 6336 patients and results of a survey. Ann Surg 2004; 240: 205-13.

23.- Clavien P A, Barkun J, de Oliveira M L, Vauthey J N, Dindo D, Schulick R D, et al. The Clavien-Dindo classification of surgical complications: five-year experience. Ann Surg 2009; 250: 187-96.

24.- Secchi M A, Pettinari R, Mercapide C, Bracco R, Castilla C, Cassone E, et al. Surgical management of liver hydatidosis: a multicentre series of 1412 patients. Liver Int 2010; 30: 85-93.

25.- Reza Mousavi S, Khoshnevis J, Kharazm P. Surgical treatment of hydatid cyst of the liver: drainage versus omentoplasty. Ann Hepatol 2005; 4: 272-4.

26.- Fica A, Soto A, Slater J, Peralta M, Humeres R, Castro M, et al. Clinical presentation of hydatid disease during 15 years: a case series from Santiago, Chile. Rev Chil Infectol 2012; 29: 183-91.

27.- Engin O, Calik B, Yilmaz M, Temiz E, Karagulle I. Surgical treatment of hydatid cyst. Retrospective review of 62 patients. Rev Chil
Cir 2010; 62: 114-8.

28.- Symeonidis N, Pavlidis T, Baltatzis M, Ballas K, Psarras K, Marakis G, et al. Complicated liver echinococcosis: 30 years of experience from an endemic area. Scand J Surg 2013; 102: 171-7.

29.- Yüksel O, Akyürek N, Sahin T, Salman B, Azili C, Bostanci H. Efficacy of radical surgery in preventing early local recurrence and cavityrelated complications in hydatic liver disease. J Gastrointest Surg 2008; 12: 483-9.

30.- Wagholikar G D, Sikora S S, Kumar A, Saxena R, Kapoor V K. Surgical management of complicated hydatid cysts of the liver. Trop Gastroenterol 2002; 23: 35-7.

31.- Akcan A, Sozuer E, Akyildiz H, Ozturk A, Atalay A, Yilmaz Z. Predisposing factors and surgical outcome of complicated liver hydatid cysts. World J Gastroenterol 2010; 16: 3040-8.

32.- Manterola C, Vial M, Schneeberger P, Peña J L, Hinostroza J, Sanhueza A. Precision of ELISAIgE and ELISA-IgG determination in the postoperative follow-up of patients with hepatic echinococcosis. Cir Esp 2007; 81:23-7.

33.- Chautems R, Bühler L H, Gold B, Giostra E, Poletti P, Chilcott M, et al. Surgical management and long-term outcome of complicated liver hydatid cysts caused by Echinococcus granulosus. Surgery 2005; 137: 312-6.

34.- Botrugno I, Gruttadauria S, Li Petri S, Cintorino D, Spada M, Di Francesco F, et al. Complex hydatid cysts of the liver: a single center's evolving approach to surgical treatment. Am Surg 2010; 76: 1011-15. 